\title{
The efficacy of intrauterine instillation of granulocyte colony-stimulating factor in infertile women with a thin endometrium: A pilot study
}

\author{
Dayong Lee', Jae Dong Jo², Seul Ki Kim', Byung Chul Jee', Seok Hyun Kim³ \\ 'Department of Obstetrics and Gynecology, Seoul National University Bundang Hospital, Seoul National University College of Medicine, Seongnam; \\ ${ }^{2}$ Elllemedi Women Clinic, Changwon; ${ }^{3}$ Department of Obstetrics and Gynecology, Seoul National University Hospital, Seoul National University College \\ of Medicine, Seoul, Korea
}

Objective: The study aimed to investigate the efficacy of intrauterine instillation of granulocyte colony-stimulating factor (G-CSF) on the day of ovulation triggering or oocyte retrieval in infertile women with a thin endometrium.

Methods: Fifty women whose endometrial thickness (EMT) was $\leq 8 \mathrm{~mm}$ at the time of triggering during at least one previous in vitro fertilization (IVF) cycle and an index IVF cycle were selected. On the day of triggering $(n=12)$ or oocyte retrieval $(n=38), 300 \mu \mathrm{g}$ of $\mathrm{G}$-CSF was instilled into the uterine cavity.

Results: In the 50 index IVF cycles, the mean EMT was $7.2 \pm 0.6 \mathrm{~mm}$ on the triggering day and increased to $8.5 \pm 1.5 \mathrm{~mm}$ on the embryo transfer day $(p<0.001)$. The overall clinical pregnancy rate was $22.0 \%$, the implantation rate was $15.9 \%$, and the ongoing pregnancy rate was $20 \%$. The clinical pregnancy rate $(41.7 \%$ vs. $15.8 \%)$, the implantation rate $(26.7 \%$ vs. $11.7 \%)$, and the ongoing pregnancy rate $(41.7 \%$ vs. $13.2 \%)$ were higher when G-CSF was instilled on the triggering day than when it was instilled on the retrieval day, although this tendency was likewise not statistically significant. Aspects of the stimulation process and mean changes in EMT were similar in women who became pregnant and women who did not.

Conclusion: Intrauterine instillation of G-CSF enhanced endometrial development and resulted in an acceptable pregnancy rate. Instillation of G-CSF on the triggering day showed better outcomes. G-CSF instillation should be considered as a strategy for inducing endometrial growth and good pregnancy results in infertile women with a thin endometrium.

Keywords: Endometrium; Granulocyte colony-stimulating factor; In vitro fertilization

\section{Introduction}

A thin endometrium can result from intrauterine adhesions after infection or curettage, the use of oral contraceptives or clomiphene

Received: Sep 18, 2016 · Revised: Nov 14, 2016 • Accepted: Nov 21, 2016 Corresponding author: Byung Chul Jee

Department of Obstetrics and Gynecology, Seoul National University Bundang Hospital, Seoul National University College of Medicine, 82 Gumi-ro 173 beongil, Bundang-gu, Seongnam 13620, Korea

Tel: +82-31-787-7254 Fax: +82-31-787-4054 E-mail: blasto@snubh.org

This is an Open Access article distributed under the terms of the Creative Commons Attribution Non-Commercial License (http://creativecommons.org/licenses/by-nc/4.0/) which permits unrestricted non-commercial use, distribution, and reproduction in any medium, provided the original work is properly cited. citrate, congenital anomalies such as Turner syndrome, or previous radiotherapy [1]. However, a thin endometrium has occasionally been observed in some patients participating in vitro fertilization (IVF) cycles despite the absence of demonstrable causes. The incidence of thin endometrium ( $\leq 7 \mathrm{~mm}$ ) has been reported to be $2.4 \%$ in IVF cycles [2]. Thin endometrium has been suggested to be associated with poor IVF success rates. The odd ratios for achieving pregnancy through IVF in women with a thin endometrium have been reported to exhibit wide variability, ranging from 0.04 to 0.73 in women with endometrial thickness (EMT) $\leq 7 \mathrm{~mm}$ compared with women with EMT > $7 \mathrm{~mm}$ [2].

The suggested treatment strategies for unexplained thin endome- 
trium include high-dose estrogen supplementation, vaginal sildenafil, low-dose aspirin, pentoxifylline, or tocopherol. Although such medical treatments are sometimes effective, some women still do not respond. Conclusive evidence is still lacking regarding their therapeutic effects on the endometrium [1].

Intrauterine instillation of granulocyte colony-stimulating factor (GCSF) to promote endometrial development was first proposed by Gleicher et al. [3] in 2011. In four women with persistent thin endometrium (3-6.2 mm) despite conventional therapies including estrogen and a vasodilator, $300 \mu \mathrm{g}$ of G-CSF was instilled into the uterine cavity 2 to 9 days before embryo transfer [3]. All endometria developed to be thicker than $7 \mathrm{~mm}$ (range, 7.3-10.2 mm), and embryo transfer was therefore attempted. All four patients conceived thereafter, but an intramural ectopic pregnancy occurred in one woman. In a subsequent prospective cohort study by the same authors, 21 women who showed EMT $<7 \mathrm{~mm}$ at the time of triggering in previous IVF cycle(s) were recruited and $300 \mu \mathrm{g}$ of G-CSF was instilled into the uterine cavity 6 to 12 hours before human chorionic gonadotropin (hCG) triggering in the next IVF cycle [4]. The endometria became thicker than $7 \mathrm{~mm}$ in all women, the mean EMT increased from 6.4 $\mathrm{mm}$ before instillation to $9.3 \mathrm{~mm}$ at the time of embryo transfer, and pregnancy was achieved in four patients (19.1\%).

In a subsequent study, $300 \mu \mathrm{g}$ of G-CSF was instilled in the same manner as in Gleicher et al's report [4] (6-12 hours before hCG triggering) in 37 women who showed an EMT $<7 \mathrm{~mm}$ at the time of triggering in previous IVF cycles [5]. The mean EMT was $6.7 \mathrm{~mm}$ before infusion and increased to $8.4 \mathrm{~mm}$ after infusion; the pregnancy rate was $18.9 \%$.

Three studies have been performed of G-CSF instillation in fresh IVF cycles in women with a thin endometrium and have reported relatively favorable pregnancy rates; however, no well-designed randomized controlled trials have been published [3-5]. Therefore, the effectiveness of G-CSF instillation for infertile women with a thin endometrium has not been conclusively established. Furthermore, it is still unknown whether G-CSF should be instilled on the triggering day or the oocyte retrieval day and whether G-CSF instillation is beneficial in specific groups. In the initial study by Gleicher et al. [3], GCSF was instilled 2 to 9 days before embryo transfer. In subsequent studies, G-CSF was instilled 6 to 12 hours before triggering $[4,5]$.

In our center, G-CSF was usually instilled on the morning of the triggering day or immediately after oocyte retrieval according to the clinician's preference. Herein, we report our experience with the intrauterine instillation of G-CSF in women with a persistently thin endometrium in a fresh IVF cycle. We compared endometrial changes and pregnancy outcomes according to whether G-CSF was instilled on the triggering day or on the oocyte retrieval day.

\section{Methods}

\section{Subjects}

A retrospective study was performed after receiving approval from the Institutional Review Board (IRB) of Ellemedi Women's Clinic, Changwon, Korea (IRB no. EOG-2016-02). We selected 50 women with an EMT $\leq 8 \mathrm{~mm}$ at the time of hCG triggering during at least one previous IVF cycle and an index IVF cycle. We informed all women about the off-label use of G-CSF and only included participants who consented to this study design. All index IVF cycles were performed from May 2015 to May 2016.

All women underwent diagnostic hysteroscopy prior to an index IVF cycle, and intrauterine synechiae were absent in all women. Endometrial polyps were identified and removed in 18 women. Thus, no participants had uterine or endometrial abnormalities except for a thin endometrium at the time of the index IVF cycle. Vaginal sildenafil was attempted in five women prior to an index IVF cycle, but no endometrial improvement occurred.

The mean age of the participants was 38.2 \pm 4.2 years (range, $29-47$ years), and age of their husbands was 38.0 \pm 4.2 years (range, 29-45 years) at the time of the index IVF cycle. The participants did not have any history of endocrine disorders or systemic diseases such as chronic neutropenia, sickle cell disease, or renal disease. Twenty-four women had at least one child. The indications for IVF were unexplained infertility $(n=18)$, decreased ovarian reserve or age factor infertility $(n=16)$, tubal infertility $(n=10)$, male factor infertility $(n=4)$, endometriosis $(n=1)$, and polycystic ovary syndrome $(n=1)$.

\section{IVF protocol and G-CSF instillation}

Controlled ovarian stimulation was performed using urinary or recombinant follicle-stimulating hormone (FSH), with or without letrozole, beginning on day 3 of the menstrual cycle. The pituitary was suppressed by a gonadotropin-releasing hormone $(\mathrm{GnRH})$ antagonist protocol $(n=27)$ or a luteal long protocol of $\mathrm{GnRH}$ agonist $(n=19)$. A modified natural IVF cycle without pituitary suppression was performed in four women. When dominant follicles averaged 19 $\mathrm{mm}$ in diameter, ovulation was triggered by a dual method (GnRH agonist and low-dose $\mathrm{hCG}(\mathrm{n}=39)$, urinary or recombinant $\mathrm{hCG}$ $(n=10)$, or a $\mathrm{GnRH}$ agonist protocol $(n=1)$. Ultrasound-guided retrieval of oocytes was performed 35 to 36 hours later.

Sperm quality was defined as normal $(n=41)$ when the semen parameters were within the World Health Organization (WHO) reference values regardless of the patient's diagnosis. Samples with parameters outside the WHO criteria, but not requiring intracytoplasmic sperm injection (ICSI), were classified as subnormal, while abnormal sperm samples $(n=6)$ were defined as those that required ICSI.

G-CSF (300 $\mu \mathrm{g} / 1 \mathrm{~mL}$ of Leucostim; Dong-A Pharmaceutical, Seoul, 
Korea) was administered into the uterine cavity by using a soft embryo transfer catheter on the morning of the triggering day (9-12 hours before hCG triggering) $(n=12)$ or immediately after oocyte retrieval $(n=38)$. The embryos were transferred 3 days $(n=41)$ or 5 days $(n=9)$ after oocyte retrieval. EMT was measured both on the morning of the triggering day and just before embryo transfer. Luteal phase support was performed through vaginal progesterone (100 mg of Lutinus twice a day; Ferring Pharmaceuticals, Saint-Prex, Switzerland) from the oocyte retrieval day until 9 weeks of gestation. Clinical pregnancy was defined as the presence of at least one intrauterine gestational sac (GS). Ongoing pregnancy was defined as a pregnancy that continued over 12 weeks of gestational age. Clinical miscarriage was defined if the pregnancy terminated before 12 weeks of gestational age.

\section{Statistical analysis}

The changes in EMT and the outcomes of IVF were compared between the groups of intrauterine instillation of G-CSF on the day of ovulation triggering or oocyte retrieval. The outcomes of IVF were compared between day 3 and day 5 transfer cycles, and between pregnant and non-pregnant cycles. The cycles with EMT $7 \mathrm{~mm}$ or less versus greater than $7 \mathrm{~mm}$ on the day of triggering were compared, either. Statistical analysis was performed using SPSS ver. 22.0 (IBM Co., Armonk, NY, USA). The parametric Student's $t$-test or the nonparametric Mann-Whitney $U$ test was used to compare the means. The data were presented as mean \pm standard deviation and nonparametric data were presented as median (95\% confidence interval). The chi-square test or the Fisher exact test was used to compare proportions, as appropriate. The $p$-values $<0.05$ were considered to indicate statistical significance.

Table 1. Clinical characteristics and stimulation outcomes according to whether G-CSF was instilled on the triggering or oocyte retrieval day

\begin{tabular}{|c|c|c|c|}
\hline Variable & Triggering day $(n=12)$ & Oocyte retrieval day $(n=38)$ & $p$-value \\
\hline \multicolumn{4}{|l|}{ Age (yr) } \\
\hline Female & $34.5(31.5-38.8)$ & $39.4(38.0-40.8)$ & 0.001 \\
\hline Male & $36.5(34.0-41.0)$ & $39.0(35.0-40.0)$ & NS \\
\hline Parous women & 5 & 19 & NS \\
\hline Body mass index (kg/m²) & $21.4(19.4-24.3)$ & $21.4(20.6-22.5)$ & NS \\
\hline Indications for IVF & & & NS \\
\hline Unexplained & 7 & 11 & \\
\hline Decreased ovarian reserve & 2 & 14 & \\
\hline Tubal & 0 & 10 & \\
\hline Male & 3 & 1 & \\
\hline Endometriosis & 0 & 1 & \\
\hline Polycystic ovary syndrome & 0 & 1 & \\
\hline Previous IVF cycle & $3(1-4)$ & $2(1-3)$ & NS \\
\hline Basal serum FSH (IU/L) & $7.5(6.0-10.0)$ & $8.5(8.0-11.0)$ & NS \\
\hline Random serum AMH (ng/mL) & $2.2(0.6-5.2)$ & $1.4(0.8-2.3)$ & NS \\
\hline Ovarian stimulation & & & NS \\
\hline Modified natural & 1 & 3 & \\
\hline Gonadotropin & 6 & 25 & \\
\hline Letrozole+gonadotropin & 5 & 10 & \\
\hline Pituitary suppression & & & NS \\
\hline GnRH antagonist & 6 & 21 & \\
\hline GnRH agonist & 5 & 14 & \\
\hline None & 1 & 3 & \\
\hline Triggering & & & NS \\
\hline GnRH agonist+low-dose hCG & 27 & 12 & \\
\hline $\mathrm{hCG}$ & 10 & 0 & \\
\hline GnRH agonist & 1 & 0 & \\
\hline Serum $E_{2}$ at triggering day $(\mathrm{pg} / \mathrm{mL})$ & $868(88-2,822)$ & $1,117(430-1,530)$ & NS \\
\hline No. of mature oocytes & $7(3-11)$ & $5(3-6)$ & NS \\
\hline Sperm & & & 0.024 \\
\hline Normal & 7 & 34 & \\
\hline Subnormal & 1 & 2 & \\
\hline Abnormal & 4 & 2 & \\
\hline Fertilization rate (\%) & $69(43-100)$ & $76(60-80)$ & NS \\
\hline
\end{tabular}

Values are presented as median (95\% confidence interval).

G-CSF, granulocyte colony-stimulating factor; NS, not significant; IVF, in vitro fertilization; FSH, follicle-stimulating hormone; AMH, anti-Müllerian hormone; $\mathrm{GnRH}$, gonadotropin-releasing factor; $\mathrm{hCG}$, human chorionic gonadotropin; $\mathrm{E}_{2}$, estradiol. 


\section{Results}

In the 50 subjects, the mean EMT in the previous failed IVF cycle(s) was $7.6 \pm 1.1 \mathrm{~mm}$. In the 50 index IVF cycles, the mean EMT on the triggering day was $7.2 \pm 0.6 \mathrm{~mm}$ (range, 5.4-7.9 mm). After G-CSF instillation on either the triggering or oocyte retrieval day, the mean EMT on the embryo transfer day significantly increased to $8.5 \pm 1.5$ $\mathrm{mm}$ (range, 6.0-12.0 mm) ( $p<0.001)$. The EMT change from triggering to embryo transfer day ranged from $-16.7 \%$ to $86.4 \%$ (mean, $20.5 \% \pm 23.7 \%)$.

In the 50 index IVF cycles, the serum hCG test was initially positive in 14 women, but a chemical pregnancy was identified in one woman and a tubal ectopic pregnancy occurred in two women. In 11 women, at least one intrauterine GS was identified (two GSs in six women). The overall clinical pregnancy rate per transfer was $22.0 \%$ (11 of 50) and the twinning rate was $54.5 \%$ (6 of 11). The overall implantation rate per transferred embryo was $15.9 \%$ (17 of 107). One miscarriage occurred in one woman who showed one intrauterine GS after transfer on day 3, making the ongoing pregnancy rate $20 \%$ (10 of 50$)$.

The clinical pregnancy rate (24.4\% [10 of 41] vs. $11.1 \%$ [1 of 9]), implantation rate (16.9\% [15 of 89] vs. $11.1 \%$ [2 of 18]), and ongoing pregnancy rate (22.0\% [9 of 41 ] vs. $11.1 \%$ [ 1 of 9 ]) tended to be higher in day 3 transfer cycles than day 5 transfer cycles, but this trend was not statistically significant.

The subjects in whom G-CSF was installed on the triggering day were younger and the sperm quality was inferior in comparison to those in whom G-CSF was instilled on the oocyte retrieval day, but the serum levels of basal FSH, random anti-Müllerian hormone, and estradiol on triggering day were similar (Table 1). The number of mature oocytes retrieved and the fertilization rate was also similar between the two groups (Table 1). The rates of clinical pregnancy, implantation, miscarriage, and ongoing pregnancy were all similar between the two groups (Table 2). The mean EMT on the triggering day or on the embryo transfer day was similar between the two groups, as was the EMT change, although the mean EMT significantly increased from the triggering day to the embryo transfer day in each group (Table 2).

When all parameters were compared between pregnant $(n=11)$ and non-pregnant women $(n=39)$, none of the parameters showed a difference (data not shown). The EMT significantly increased from $7.5 \mathrm{~mm}$ (range, $7.0-7.8 \mathrm{~mm}$ ) to $8.8 \mathrm{~mm}$ (range, 7.0-10.0 $\mathrm{mm}$ ) in the pregnant group $(p=0.041)$ and from $7.4 \mathrm{~mm}$ (range, 7.1-7.5 $\mathrm{mm}$ ) to $8.0 \mathrm{~mm}$ (range, 7.4-9.5 mm) in the non-pregnant group $(p<0.001)$. The EMT change was similar in both groups (21.1\% [range, 6.7\%$50.0 \%$ ] vs. $26.9 \%$ [range, $12.7 \%-35.1 \%$ ], $p=0.755$ ). Interestingly, despite G-CSF instillation, the EMT decreased in seven women, among whom one woman conceived despite her EMT changing from 7.5 $\mathrm{mm}$ to $7 \mathrm{~mm}$.

Table 2. EMT and pregnancy outcomes according to whether G-CSF was instilled on the triggering or oocyte retrieval day

\begin{tabular}{|c|c|c|c|}
\hline Variable & Triggering day $(n=12)$ & Oocyte retrieval day $(n=38)$ & $p$-value \\
\hline EMT at triggering day (mm) & $7.5(6.0-7.6)$ & $7.4(7.1-7.5)$ & NS \\
\hline EMT at embryo transfer day (mm) & $8.5(7.4-9.0)$ & $8.0(7.3-9.5)$ & NS \\
\hline Change of EMT (\%) & $20.0(5.3-50.0)^{a)}$ & $26.6(12.7-35.1)^{a)}$ & NS \\
\hline No. of embryo transfer cycles & & & NS \\
\hline Day 3 transfer & 8 & 33 & \\
\hline Day 5 transfer & 4 & 5 & \\
\hline \multicolumn{4}{|l|}{ No. of embryos transferred } \\
\hline Day 3 transfer & $3(2-3)$ & $2(1-3)$ & NS \\
\hline Day 5 transfer & $2.5(1-3)$ & $2(1-3)$ & NS \\
\hline \multicolumn{4}{|l|}{ Highest-quality embryo (day 3 only) } \\
\hline A & $1(3.3)$ & $12(15.6)$ & NS \\
\hline$A+B$ & $10(33.3)$ & $36(46.8)$ & NS \\
\hline Clinical pregnancy & $5(41.7)$ & $6(15.8)$ & NS \\
\hline Day 3 transfer & $4(50.0)$ & $6(18.2)$ & NS \\
\hline Day 5 transfer & $1(25.0)$ & 0 & NS \\
\hline Clinical miscarriage & 0 & $1(2.6)$ & NS \\
\hline Ongoing pregnancy & $5(41.7)$ & $5(13.2)$ & NS \\
\hline \multicolumn{4}{|l|}{ Implantation rate $(\%, \mathrm{n})$} \\
\hline Day 3 transfer & $28.6(6 / 21)$ & $13.2(9 / 68)$ & NS \\
\hline Day 5 transfer & $22.2(2 / 9)$ & $0(0 / 9)$ & NS \\
\hline
\end{tabular}

Values are presented as median ( $95 \%$ confidence interval) or number (\%) unless otherwise indicated.

EMT, endometrial thickness; G-CSF, granulocyte colony-stimulating factor; NS, not significant.

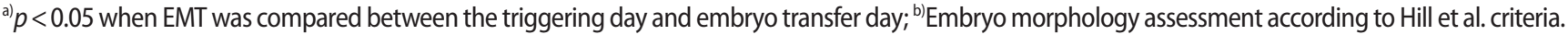


Table 3. Pregnancy outcomes according to whether EMT on the triggering day was $\leq 7 \mathrm{~mm}$ or $>7 \mathrm{~mm}$

\begin{tabular}{|c|c|c|c|}
\hline Variable & $\leq 7 \mathrm{~mm}(\mathrm{n}=14)$ & $>7 \mathrm{~mm}(\mathrm{n}=36)$ & $p$-value \\
\hline EMT at triggering day $(\mathrm{mm})$ & $6.3(5.9-7.0)$ & $7.5(7.4-7.6)$ & \\
\hline EMT at embryo transfer day (mm) & $8.0(7.0-11.0)$ & $8.4(7.7-9.5)$ & NS \\
\hline Change of EMT (\%) & $30.5(6.1-71.4)^{\mathrm{a})}$ & $20.0(6.7-29.9)^{\mathrm{a})}$ & 0.004 \\
\hline G-CSF instillation & & & NS \\
\hline Triggering day & 4 & 8 & \\
\hline Oocyte retrieval day & 9 & 28 & \\
\hline No. of embryo transfer cycles & & & NS \\
\hline Day 3 transfer & 10 & 31 & \\
\hline Day 5 transfer & 4 & 5 & \\
\hline Clinical pregnancy & $2(14.3)$ & $9(25.0)$ & NS \\
\hline Day 3 transfer & $2(20.0)$ & $8(25.8)$ & \\
\hline Day 5 transfer & 0 & $1(20.0)$ & \\
\hline Clinical miscarriage & 0 & $1(2.8)$ & NS \\
\hline Ongoing pregnancy & $2(14.3)$ & $8(22.2)$ & NS \\
\hline Implantation rate $(\%, \mathrm{n})$ & $12.1(4 / 33)$ & $17.6(13 / 74)$ & NS \\
\hline Day 3 transfer & $16.0(4 / 25)$ & $17.2(11 / 64)$ & \\
\hline Day 5 transfer & $0(0 / 8)$ & $20.0(2 / 10)$ & \\
\hline
\end{tabular}

Values are presented as median (95\% confidence interval) or number (\%) unless otherwise indicated.

EMT, endometrial thickness; NS, not significant; G-CSF, granulocyte colony-stimulating factor .

a) $p<0.05$ when EMT was compared between the triggering day and the embryo transfer day.

In women with an EMT $\leq 7 \mathrm{~mm}$ on the triggering day, the mean EMT change was significantly greater than in women with an EMT $>7 \mathrm{~mm}$ on the triggering day $(p=0.004)$ (Table 3$)$. However, the rates of clinical pregnancy, implantation, miscarriage, and ongoing pregnancy were all similar between the two groups.

\section{Discussion}

In the present study, intrauterine instillation of G-CSF resulted in an acceptable pregnancy rate in women with a consistently thin endometrium, as in two previous studies [4,5]. EMT improved after G-CSF instillation from the triggering day to the embryo transfer day, except in seven women. Unlike in other studies, we instilled G-CSF on the triggering day or the oocyte retrieval day; for the first time, we demonstrated a similar pregnancy outcome between these two instillation groups. However, pregnancy outcomes tended to be higher in the group in which instillation was performed on the triggering day. Therefore, G-CSF instillation on the triggering day may be associated with a better prognosis.

Unlike other studies, our study included nine cycles where embryo transfer was performed on day 5 . Since the pregnancy rate associated with day 5 transfer is known to be higher than that associated with day 3 transfer, day 5 transfer is commonly applied in women with a thin endometrium [6]. Nonetheless, the pregnancy and implantation rates associated with day 5 transfer were lower than expected in the present study. Given this information, we suggest that day 3 transfer may also lead to a good prognosis in women with a thin endometrium who undergo G-CSF instillation.

Unlike the study conducted by Gleicher et al. [4], EMT did not increase in all women after G-CSF instillation in our study. However, as in the previous two studies, no difference in EMT gain was found between women who conceived and those who did not. Interestingly, a reduced EMT despite G-CSF instillation was found in seven women, one of whom conceived. She received G-CSF on the triggering day, and her EMT was $7.5 \mathrm{~mm}$ on the triggering day and $7.0 \mathrm{~mm}$ on the transfer day. After two embryos were transferred on day 5 , she conceived twins.

In the present study, we defined a thin endometrium as having an EMT $\leq 8 \mathrm{~mm}$. In fact, controversy exists regarding the definition of so-called thin endometrium and the minimally required EMT for successful pregnancy. Various investigators have advocated $\geq 7 \mathrm{~mm}$, $\geq 8 \mathrm{~mm}$, and $\geq 9 \mathrm{~mm}$ as cutoffs predictive of successful pregnancies $[2,7]$. Nonetheless, when we analyzed 14 women with an EMT $\leq 7$ $\mathrm{mm}$ on the triggering day in the present study, the intrauterine instillation of G-CSF resulted in an acceptable pregnancy rate (14.3\% overall and $20 \%$ in the day 3 transfer group). In the present study, the lowest EMT was $7 \mathrm{~mm}$ on the triggering day among patients who conceived (range, 7-10 mm), while no pregnancy occurred in women with EMT $<7 \mathrm{~mm}$ on the triggering day despite G-CSF instillation.

Various mechanisms have been suggested for the inability of embryos to be implanted in a thin endometrium. Endometrial tissue is composed of basal and functional layers. The basal layer consists of 
large spiral arteries and is maintained throughout the menstrual cycle, whereas the functional layer includes relatively fine spiral arteries and changes throughout the menstrual cycle. After ovulation, the spiral arteries within the functional layer shrink, causing oxygen tension to decrease in the functional layer, which supports a favorable environment for embryo implantation [8]. If the functional layer is inadequately developed, embryos are close to the large spiral arteries within the basal layer; thus, embryos are exposed to a highly oxygenated environment. At this time, increased levels of reactive oxygen species can compromise embryo development and implantation $[9,10]$.

The mechanisms underlying the favorable impact of G-CSF instillation on EMT and embryo implantation are still unknown. It is less likely that improved EMT alone contributes to enhanced embryo implantation, because the EMT gains after G-CSF instillation were similar in women who conceived and those who did not.

G-CSF is a kind of cytokine, first purified in mice and cloned in humans later [11]. G-CSF is a primarily hematopoietic-lineage cytokine produced by diverse cells such as bone marrow cells, stromal cells, fibroblasts, endothelial cells, monocytes, and macrophages. The human endometrium also expresses G-CSF mRNA throughout the menstrual cycle [12]. G-CSF acts to stimulate the proliferation and differentiation of hematopoietic stem and progenitor cells and mesenchymal precursor cells in the bone marrow $[13,14]$. It also acts to induce the recruitment and homing of mesenchymal stem-like cells into vascular injured sites $[15,16]$. The human endometrium also contains intrinsic mesenchymal stem-like cells, which possibley contribute to endometrial cyclic proliferation $[15,17]$. G-CSF is thought to stimulate intrinsic mesenchymal stem-like cells or induce the homing of extrinsic stem cells, but direct evidence is still lacking.

Receptors for G-CSF are present not only in hematopoietic lineage cells, but also in cells contributing to reproduction, such as luteinized granulosa cells, trophoblastic cells, and the endometrium [18-21]. GCSF enhances the cyclic adenosine monophosphate-mediated decidualization of human endometrial stromal cells in an autocrine or paracrine fashion [22]. It has been reported that G-CSF, when injected subcutaneously, acts on embryo implantation by modulating the immune reaction and affecting the function of macrophages in the decidua $[23,24]$. Culture of luteal endometrial tissue together with recombinant G-CSF was found to stimulate the expression of several genes that play a crucial role in embryo implantation (i.e., endometrial vascular remodeling, immune modulation, and/or cellular adhesion pathways) [25]. In patients with repeated implantation failure, decreased expression of G-CSF receptors was found in the middle luteal phase [21]. Collectively, those findings suggest that G-CSF can modulate certain endometrial changes through its receptor and thus contribute to endometrial regeneration [26]. Nonetheless, we still do not know whether extrinsic supplementation of G-CSF can induce endometrial cell proliferation and/or immune modulation within the endometrium.

One limitation of this study was that it had a relatively small sample population. The study did not have an appropriate control group and was performed in only one infertility center. The pregnancies were followed until 12 weeks of gestation, so further study would be needed to evaluate the live birth rate as a final outcome in a larger population.

Herein, we describe the efficacy of intrauterine instillation of G-CSF in women with a thin endometrium. We suggest that G-CSF instillation on the triggering day may be preferable to enhance the pregnancy and implantation rate in women with a thin endometrium. Improving endometrial growth in women with a thin endometrium is a challenge for infertility clinicians. In this aspect, G-CSF instillation should be considered as a strategy to induce adequate endometrial growth and good pregnancy results. The effectiveness of G-CSF instillation should further be confirmed via well-designed and controlled studies. Furthermore, basic research on how G-CSF can modulate endometrial growth and/or receptivity should be undertaken.

\section{Conflict of interest}

No potential conflict of interest relevant to this article was reported.

\section{References}

1. Senturk LM, Erel CT. Thin endometrium in assisted reproductive technology. Curr Opin Obstet Gynecol 2008;20:221-8.

2. Kasius A, Smit JG, Torrance HL, Eijkemans MJ, Mol BW, Opmeer $B C$, et al. Endometrial thickness and pregnancy rates after IVF: a systematic review and meta-analysis. Hum Reprod Update 2014;20:530-41.

3. Gleicher N, Vidali A, Barad DH. Successful treatment of unresponsive thin endometrium. Fertil Steril 2011;95:2123.

4. Gleicher N, Kim A, Michaeli T, Lee HJ, Shohat-Tal A, Lazzaroni E, et al. A pilot cohort study of granulocyte colony-stimulating factor in the treatment of unresponsive thin endometrium resistant to standard therapies. Hum Reprod 2013;28:172-7.

5. Kunicki M, Lukaszuk K, Woclawek-Potocka I, Liss J, Kulwikowska P, Szczyptanska J. Evaluation of granulocyte colony-stimulating factor effects on treatment-resistant thin endometrium in women undergoing in vitro fertilization. Biomed Res Int 2014;2014: 913235.

6. Glujovsky D, Farquhar C, Quinteiro Retamar AM, Alvarez Sedo CR, Blake D. Cleavage stage versus blastocyst stage embryo transfer in assisted reproductive technology. Cochrane Database Syst 
Rev 2016;(6):CD002118.

7. Shufaro Y, Simon A, Laufer N, Fatum M. Thin unresponsive endometrium: a possible complication of surgical curettage compromising ART outcome. J Assist Reprod Genet 2008;25:421-5.

8. Rossman I, Bartelmez GW. The injection of the blood vascular system of the uterus. Anat Rec 1957;128:223-31.

9. Catt JW, Henman M. Toxic effects of oxygen on human embryo development. Hum Reprod 2000;15 Suppl 2:199-206.

10. Yang HW, Hwang KJ, Kwon HC, Kim HS, Choi KW, Oh KS. Detection of reactive oxygen species (ROS) and apoptosis in human fragmented embryos. Hum Reprod 1998;13:998-1002.

11. Nagata S, Tsuchiya M, Asano S, Kaziro Y, Yamazaki T, Yamamoto O, et al. Molecular cloning and expression of cDNA for human granulocyte colony-stimulating factor. Nature 1986;319:415-8.

12. Fahey JV, Schaefer TM, Channon JY, Wira CR. Secretion of cytokines and chemokines by polarized human epithelial cells from the female reproductive tract. Hum Reprod 2005;20:1439-46.

13. Thomas J, Liu F, Link DC. Mechanisms of mobilization of hematopoietic progenitors with granulocyte colony-stimulating factor. Curr Opin Hematol 2002;9:183-9.

14. Brouard N, Driessen R, Short B, Simmons PJ. G-CSF increases mesenchymal precursor cell numbers in the bone marrow via an indirect mechanism involving osteoclast-mediated bone resorption. Stem Cell Res 2010;5:65-75.

15. Chan RW, Schwab KE, Gargett CE. Clonogenicity of human endometrial epithelial and stromal cells. Biol Reprod 2004;70:173850.

16. Singh P, Hu P, Hoggatt J, Moh A, Pelus LM. Expansion of bone marrow neutrophils following G-CSF administration in mice results in osteolineage cell apoptosis and mobilization of hematopoietic stem and progenitor cells. Leukemia 2012;26:2375-83.

17. Schwab KE, Gargett CE. Co-expression of two perivascular cell markers isolates mesenchymal stem-like cells from human endometrium. Hum Reprod 2007;22:2903-11.
18. Uzumaki H, Okabe T, Sasaki N, Hagiwara K, Takaku F, Tobita M, et al. Identification and characterization of receptors for granulocyte colony-stimulating factor on human placenta and trophoblastic cells. Proc Natl Acad Sci U S A 1989;86:9323-6.

19. Salmassi A, Schmutzler AG, Huang L, Hedderich J, Jonat W, Mettler $L$. Detection of granulocyte colony-stimulating factor and its receptor in human follicular luteinized granulosa cells. Fertil Steril 2004;81 Suppl 1:786-91.

20. Salmassi A, Schmutzler AG, Schaefer S, Koch K, Hedderich J, Jonat $W$, et al. Is granulocyte colony-stimulating factor level predictive for human IVF outcome? Hum Reprod 2005;20:2434-40.

21. Ledee N, Munaut C, Aubert J, Serazin V, Rahmati M, Chaouat G, et al. Specific and extensive endometrial deregulation is present before conception in IVF/ICSI repeated implantation failures (IF) or recurrent miscarriages. J Pathol 2011;225:554-64.

22. Tanaka T, Miyama M, Masuda M, Mizuno K, Sakamoto T, Umesaki $\mathrm{N}$, et al. Production and physiological function of granulocyte colony-stimulating factor in non-pregnant human endometrial stromal cells. Gynecol Endocrinol 2000;14:399-404.

23. Scarpellini F, Sbracia M. Use of granulocyte colony-stimulating factor for the treatment of unexplained recurrent miscarriage: a randomised controlled trial. Hum Reprod 2009;24:2703-8.

24. Santjohanser C, Knieper C, Franz C, Hirv K, Meri O, Schleyer M, et al. Granulocyte-colony stimulating factor as treatment option in patients with recurrent miscarriage. Arch Immunol Ther Exp (Warsz) 2013;61:159-64.

25. Rahmati M, Petitbarat M, Dubanchet S, Bensussan A, Chaouat G, Ledee N. Granulocyte-colony stimulating factor related pathways tested on an endometrial ex-vivo model. PLoS One 2014;9: e102286.

26. Zhao J, Tian T, Zhang Q, Wang Y, Li Y. Use of granulocyte colonystimulating factor for the treatment of thin endometrium in experimental rats. PLoS One 2013;8:e82375. 